\title{
BMJ Open Effect of physical exercise on cognitive function and brain measures after chemotherapy in patients with breast cancer (PAM study): protocol of a randomised controlled trial
}

\author{
Lenja Witlox, ${ }^{\oplus 1}$ Sanne B Schagen, ${ }^{2}$ Michiel B de Ruiter, ${ }^{2}$ Mirjam I Geerlings, ${ }^{1}$ \\ Petra H M Peeters, ${ }^{1}$ Emmie W Koevoets, ${ }^{1,2}$ Elsken van der Wall, ${ }^{3}$ Martijn Stuiver, ${ }^{4,5}$ \\ Gabe Sonke, ${ }^{4}$ Miranda J Velthuis, ${ }^{6}$ Job A M van der Palen, ${ }^{7,8}$ Jan J Jobsen, ${ }^{7}$ \\ Anne M May, ${ }^{1}$ E M Monninkhof ${ }^{1}$
}

To cite: Witlox L, Schagen SB, de Ruiter MB, et al. Effect of physical exercise on cognitive function and brain measures after chemotherapy in patients with breast cancer (PAM study): protocol of a randomised controlled trial. BMJ Open 2019;9:e028117. doi:10.1136/ bmjopen-2018-028117

- Prepublication history for this paper is available online. To view these files, please visit the journal online (http://dx.doi. org/10.1136/bmjopen-2018028117).

Received 22 November 2018 Revised 1 March 2019 Accepted 11 April 2019

D) Check for updates

(C) Author(s) (or their employer(s)) 2019. Re-use permitted under CC BY-NC. No commercial re-use. See rights and permissions. Published by BMJ.

For numbered affiliations see end of article.

Correspondence to Dr E M Monninkhof; e.monninkhof@umcutrecht.nl

\section{ABSTRACT}

Introduction After treatment with chemotherapy, many patients with breast cancer experience cognitive problems. While limited interventions are available to improve cognitive functioning, physical exercise showed positive effects in healthy older adults and people with mild cognitive impairment. The Physical Activity and Memory study aims to investigate the effect of physical exercise on cognitive functioning and brain measures in chemotherapy-exposed patients with breast cancer with cognitive problems.

Methods and analytics One hundred and eighty patients with breast cancer with cognitive problems $2-4$ years after diagnosis are randomised (1:1) into an exercise intervention or a control group. The 6-month exercise intervention consists of twice a week 1-hour aerobic and strength exercises supervised by a physiotherapist and twice a week 1-hour Nordic or power walking. The control group is asked to maintain their habitual activity pattern during 6 months. The primary outcome (verbal learning) is measured at baseline and 6 months. Further measurements include online neuropsychological tests, self-reported cognitive complaints, a 3-tesla brain MRI, patient-reported outcomes (quality of life, fatigue, depression, anxiety, work performance), blood sampling and physical fitness. The MRI scans and blood sampling will be used to gain insight into underlying mechanisms. At 18 months online neuropsychological tests, self-reported cognitive complaints and patient-reported outcomes will be repeated.

Ethics and dissemination Study results may impact usual care if physical exercise improves cognitive functioning for breast cancer survivors.

Trial registration number NTR6104

\section{INTRODUCTION}

Increased survival of patients with breast cancer has led to more emphasis on studying long-term consequences of treatment. Research has shown that a portion

\section{Strengths and limitations of this study}

- Since only small pilot studies have been conducted, the Physical Activity and Memory study is the first large and sufficiently powered randomised controlled trial examining the effect of physical exercise on cognitive functioning and brain measures in breast cancer survivors.

- The study is designed to investigate the effect of exercise and aims to provide insight into the underlying mechanisms.

- Patients with self-reported cognitive problems confirmed by neuropsychological tests are included in the study.

- The present study might hamper generalisability to a broader patient population who experience cognitive problems after cancer.

of patients with breast cancer experience cognitive decline after cancer treatment. The observed cognitive changes frequently concern learning and memory functioning, speed of information processing and executive functioning. ${ }^{12}$ The number of affected patients varies between studies from $18 \%$ to $60 \%$ following cancer diagnoses and treatment with chemotherapy. ${ }^{2-4}$ This wide range is largely explained by differences in timing of assessment, criteria held for cognitive decline and treatment characteristics. ${ }^{5}$ Cognitive problems can persist after completion of treatment. ${ }^{6}$ Even after 20 years, cognitive differences between chemotherapy-treated patients and non-cancer controls have been established. ${ }^{6} 7$ The cognitive problems are generally mild to moderate and can adversely affect work ability, interpersonal relationships and leisure activities. ${ }^{8}$ 
Chemotherapy is an important pillar in the treatment of primary breast cancer, which impairs cognition by several mechanisms. ${ }^{2}$ Preclinical studies showed that chemotherapy agents can disrupt various neurobiological processes, which can lead to cognitive impairment. Effects of cellular toxicity on cognitive impairment are described (neurons, glial cells, progenitor and stem cells), but also reduced white matter integrity and inflammatory reactions as vascular toxicity and oxidative stress. ${ }^{9-11}$ These mechanisms are not mutually exclusive and one can also influence the other.

Neuroimaging studies in patients with non-central nervous system cancer have been performed to shed light on the neural substrate of the cognitive changes in patients after diagnosis and treatment with chemotherapy. Various MRI studies have shown brain volume reductions after chemotherapy, particularly in neocortex, which is sometimes directly associated with cognitive decline. ${ }^{612-16}$ Although these reductions seem to be transient to some extent, they can persist for many years. ${ }^{16}$ MRI diffusion imaging studies found reductions in white matter microstructure, ${ }^{6} 12131718$ with some studies observing that patients who showed more severe cognitive decline also showed more intense reductions in white matter microstructure. ${ }^{19}$ Additionally, alterations in cerebral blood flow as measured with arterial spin labelling have been associated with cognitive performance in women receiving chemotherapy. ${ }^{20}$ Using functional MRI a study of Deprez et $a l^{22}$ reported decreased brain activation during multitasking of chemotherapy-treated patients, which was associated with an increase in subjective cognitive complaints.

A promising non-pharmacological intervention for cognitive problems is physical exercise. In healthy older adults and people with mild cognitive impairment, evidence of positive effects of exercise on cognition is accumulating. ${ }^{23}{ }^{24} \mathrm{~A}$ meta-analysis and also three more recent randomised controlled trials (RCTs) showed that physical activity in healthy older adults improved cognitive function in several domains including memory, ${ }^{24-26}$ executive function, ${ }^{24} 27$ attention ${ }^{24}$ and processing speed. ${ }^{24}$

Until now, little is known about the mechanisms underlying this effect. Results from animal and human studies have elucidated several mechanisms by which exercise might affect the brain during ageing. These mechanisms include neurogenesis, ${ }^{28}$ vascular changes (ie, increased oxygen saturation, the promotion of angiogenesis and increased cerebral blood flow ${ }^{29}$ ), changes in neurotransmitters (specifically catecholamine synthesis ${ }^{30}$ ) and inflammatory factors. Systematic reviews reported potential effects of exercise after completion of chemotherapy on tumour necrosis factor-alpha, ${ }^{31} \mathrm{C}$ reactive protein ${ }^{32}$ interleukin(IL)-2, IL-6 and IL8, although no consistent effects have been found. The hypothesised mechanism that is currently best supported by evidence is that exercise leads to increased levels of neurotrophic factors, which in turn have a positive influence on neurogenesis. ${ }^{33}$ Brain-derived neurotrophic factor (BDNF) has been implicated in the differentiation, extension and survival of neurons in the hippocampus, cortex, striatum and cerebellum. $^{34}{ }^{35}$ Results from a meta-analysis indicated that aerobic exercise increased resting BDNF levels. ${ }^{36}$ In animal studies, BDNF has already been shown a mediating factor of exercise effects on cognitive function. ${ }^{37}$

In addition, neuroimaging data provide insights into the neurobiological mechanisms underlying cognitive recovery by exercise. In healthy elderly and patients with mild cognitive impairment, exercise training led to a significantly increased hippocampal volume, ${ }^{38} 39$ which was associated with improvements in spatial memory. ${ }^{38}$ In another study, increased brain volume in both grey and white matter regions were reported after a 6-month aerobic fitness programme. ${ }^{40}$ Furthermore, cerebral blood flow was increased in the anterior cingulate region, which was related to a better memory performance. ${ }^{41}$

Physical exercise might also be beneficial for patients with cancer experiencing cancer-related cognitive problems. ${ }^{42}$ Two pilot studies studied the effect of exercise on cognitive functioning of patients with breast cancer after primary treatment. ${ }^{4344}$ Both studies included women with self-reported cognitive problems after chemotherapy. The 19 women in the study of Campbell et $a l^{43}$ and 87 women in the study of Hartman $e t a l^{44}$ were randomly allocated to an aerobic exercise intervention of $150 \mathrm{~min}$ per week, moderate to vigorous exercise or control group. The intervention of Campbell et al consisted of 24-week supervised and home-based aerobic exercise programme, while Hartman et al assessed a 12-week home-based aerobic exercise intervention. ${ }^{43}$ Even though the studies were small and included patients with breast cancer with cognitive problems based on self-report only which might also be influenced by other factors, processing speed in the exercise group was improved compared with the control group. ${ }^{434}$ However, Hartman et alfound this improvement only in a subgroup of patients within 2 years from breast cancer diagnosis. ${ }^{44}$ Furthermore, this study also suggested an improvement in self-reported cognition, although not statistically significant. ${ }^{44}$ In another pilot study conducted during chemotherapy $(n=17)$, the strength exercise group performed better on concentration and verbal memory than the control group. ${ }^{45}$ In addition, two larger RCTs found an indication that physical exercise during cancer treatment improved objective cognitive functioning like processing speed. ${ }^{46} 47$ Limitations of these studies are that cognition was not the primary outcome and was only measured by one neuropsychological test. Larger clinical trials are warranted to prove potential benefits of physical exercise in patients with cancer with cognitive problems.

Therefore, we designed an RCT to examine the effects of physical exercise on cognitive functioning in chemotherapy-exposed patients with breast cancer with self-reported cognitive problems confirmed by standardised and validated neuropsychological tests. We anticipate that exercise improves cognitive functioning. Neurogenesis in the hippocampus is one of the most important proposed mechanisms by which exercise might affect the brain. 


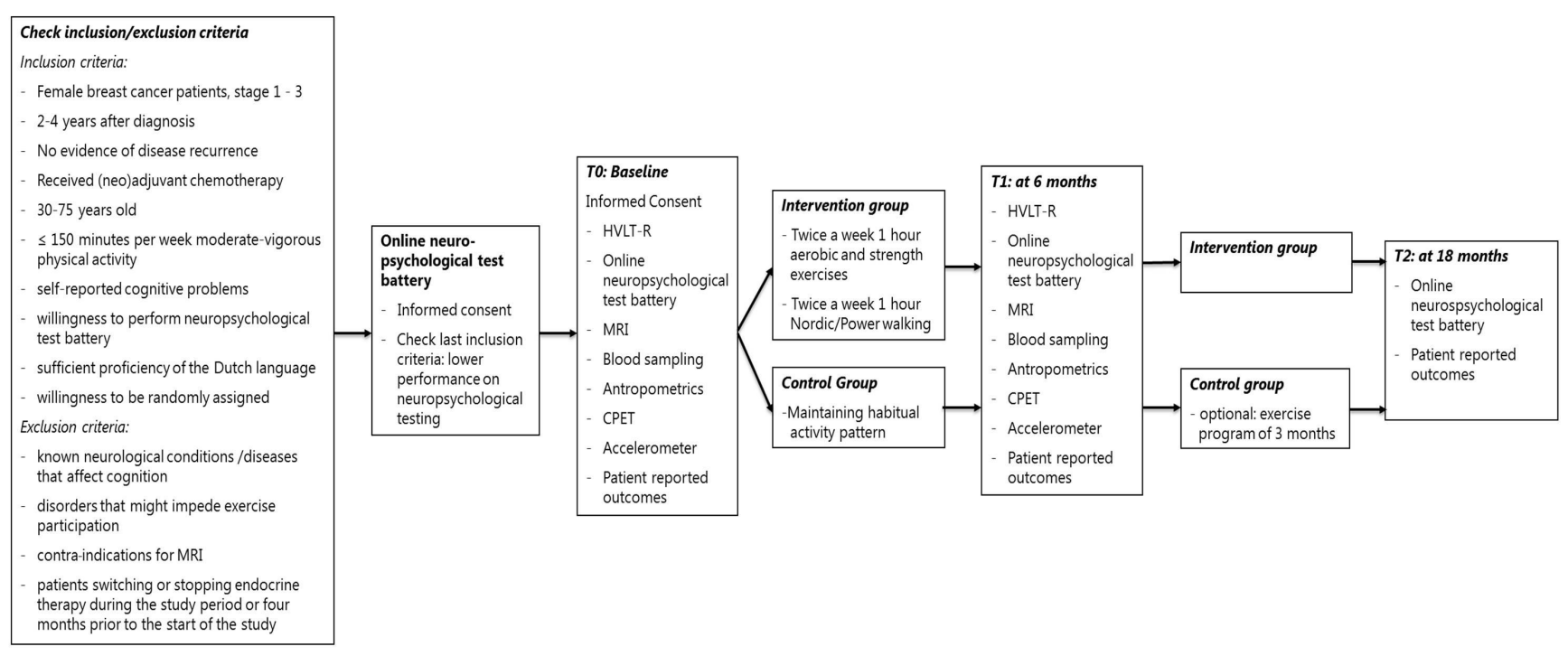

Figure 1 Overview of the PAM study. CPET, cardiopulmonary exercise test; HVLT-R, Hopkins Verbal Learning Test-Revised; PAM, Physical Activity and Memory.

Since the hippocampus is also associated with memory functioning, our primary outcome is verbal learning and memory. Furthermore, we hypothesise that exercise training will result in changes, visible on brain MRI, such as increased brain volume (including the hippocampus), increased connectivity of white matter (especially connections with the hippocampus) and increased perfusion. To study this hypothesis, we designed an RCT to examine the effects of physical exercise on cognitive functioning in chemotherapy-exposed patients with breast cancer with self-reported cognitive problems confirmed by standardised and validated neuropsychological tests.

\section{METHODS AND ANALYSIS \\ Design}

The Physical Activity and Memory (PAM) study is an RCT with two study groups, that is, a 6-month exercise intervention group and a control group (see figure 1 for an overview). Recruitment for the study started in December 2016 and still is ongoing.

\section{Participants}

In total, 180 patients will participate in the PAM study. Inclusion criteria the PAM study are: female patients with breast cancer with stages 1-3, 2-4 years after their cancer diagnosis, who received treatment with (neo)adjuvant chemotherapy, 30-75 years of age at inclusion, no evidence of disease recurrence, $\leq 150 \mathrm{~min}$ per week moderate-vigorous physical activity, self-reported cognitive problems, moderate to lower performance on neuropsychological testing, sufficient proficiency of the Dutch language and willing to be randomly assigned to one of the two study arms. Exclusion criteria are: known neurological conditions and/or diseases that affect cognition (eg, dementia, multiple sclerosis, traumatic brain injury), disorders that might impede exercise participation, contraindications for MRI and patients switching or stopping endocrine therapy during the study period or 4 months prior to the start of the study. The latter is chosen since we do not know if different endocrine therapies may differ in their potential impact on cognitive performance. ${ }^{48}$ In this explanatory study, we chose to include patients with breast cancer with self-reported cognitive problems confirmed by neuropsychological tests only, since self-reported cognitive problems might also be influenced by other factors such as distress. ${ }^{3}$

\section{Recruitment and randomisation}

Patients are recruited by their treating physician or the study team. First, the treating physician sends potential eligible candidates an invitation letter with study information. With the invitation letter, also the information brochure and study leaflet are provided. In addition, study information is shared via social media such as the Facebook page of patient organisations and funding bodies. Interested women who experience cognitive problems interfering with daily life are asked to contact the study team. Subsequently, the study team calls these women to explain further details of the study, to answer questions and to screen for inclusion and exclusion criteria. After the telephone screening, eligible women sign written informed consent specifically for performing a full online neuropsychological test battery: the Amsterdam Cognition Scan ${ }^{49}$ and sent the original written informed consent to the study team by post. The patients receive an email with instructions to conduct the test battery. ${ }^{50}$ Patients performing lower than expected, that is, a worse z-score of at least 1 compared with a control group, on at least two tests of the different domains (learning and memory, attention and working memory, processing speed, executive functioning, motor functioning) compared with the normative data of a healthy female population by 


\begin{tabular}{ll}
\hline Table 1 Content of the online test battery \\
\hline Test domain & Online test \\
\hline Learning and memory & $\begin{array}{l}\text { Wordlist learning } \\
\text { Wordlist delayed recall } \\
\text { Wordlist recognition }\end{array}$ \\
$\begin{array}{l}\text { Attention and working } \\
\text { memory }\end{array}$ & $\begin{array}{l}\text { Digit sequences I } \\
\text { Digit sequences II } \\
\text { Box tapping }\end{array}$ \\
Processing speed & $\begin{array}{l}\text { Connecting the dots I } \\
\text { Reaction time }\end{array}$ \\
Executive functioning & $\begin{array}{l}\text { Connecting the dots II } \\
\text { Place the beads }\end{array}$ \\
Motor functioning & Fill the grid
\end{tabular}

age, are eligible for inclusion. The domains and the corresponding tests are described in table 1. The cut-off z-scores are similar to cut-offs used in a previous intervention study. ${ }^{51}$

Finally, patients visit the University Medical Center (UMC) Utrecht to sign informed consent for study participation and randomisation. After baseline measurements, patients are randomly allocated via a blinding procedure of a computer-generated sequence to the intervention or control group, stratified by age (30-44, 45-59, 60-75 years) and endocrine therapy (yes or no). The randomisation programme is provided by department of data management of the Julius Center (UMC Utrecht). Enrolment and randomisation is performed by a member of the study team.

\section{EXERCISE INTERVENTION}

The 6-month exercise intervention consists of twice weekly, 1-hour sessions of aerobic and strength exercises supervised by a physiotherapist, nearby the patients' home and Nordic or power walking for 2 hours per week. The programme is tailored to the women's physical fitness level and potential constraints. The level of intensity is increased during the exercise programme. During the aerobic exercises, participants wear heart rate monitors to train on the level of intensity, as proposed in their personalised exercise protocol.

\section{Supervised aerobic and strength exercise}

The supervised exercise sessions consist of 20-25 min aerobic and 20-25 min strength training. Sessions start and end with 5-10 min warming up and cooling down. The intensity of the aerobic exercises determined by the heart rate reserve (HRR) is based on the resting heart rate and peak heart rate and resting heart rate, which are measured during the cardiopulmonary exercise test (CPET) performed at baseline.

The muscle strength exercises are performed for all major muscle groups: arms, legs, shoulders and trunk. The intensity of the strength exercises is determined by pragmatic 15 and 20 repetition maximum (RM, the maximum weight at which a muscle group can perform repetitions) tests and repeated every 4 weeks (table 2 ). A 15 and $20 \mathrm{RM}$ is assessed instead of a $1 \mathrm{RM}$ test, since this is less sensitive for injuries and easier for the physical therapist to use (without making use of calculations). A member of study team visits a training session for monitoring 1 month after the first training.

\section{Nordic or power walking}

Participants in the intervention group additionally perform twice weekly, 1 hour of Nordic or power walking at $55 \%-65 \%$ of the HRR. Patients receive Nordic walking poles and an instruction leaflet. Patients choose whether they prefer Nordic or power walking. By using poles for Nordic walking, the technique will focus more on the trunk and arms to reduce the load on the legs. ${ }^{52}$ Power walking consists of a fast walking pace including an active upper body movement. We choose these types of walking since these are more effective to increase cardiovascular fitness compared with standard walking. ${ }^{52-55}$ Besides, patients are encouraged to join supervised walking sessions to increase motivation and adherence. If walking is not possible, due to medical constraints, other exercises are allowed such as cycling (also at 55\%-65\% HRR). The

Table 2 Supervised exercise programme of the PAM study

\begin{tabular}{|c|c|c|}
\hline Week & Aerobic & Strength \\
\hline $1-4$ & $40 \%-60 \%$ HRR & \multirow[b]{2}{*}{$\begin{array}{l}\text { One circuit of 20-25 repetitions. Weights based on } \\
20 \mathrm{RM} \text { tests (repeated every } 4 \text { weeks) } \\
\text { Exercises: legs (squat, lunges, calve raises), arms } \\
\text { (biceps curl, triceps extension), shoulder (shoulder } \\
\text { press), thorax (barbell bench press), back (rowing). } \\
\text { Abdomen: crunch } 30-40 \text { repetitions. }\end{array}$} \\
\hline 5-9 & $\begin{array}{l}60 \%-70 \% \text { HRR } 15-20 \mathrm{~min} \text {, } \\
\text { Plus } 70 \%-89 \% \text { HRR 5-10 min. }\end{array}$ & \\
\hline $18-26$ & $\begin{array}{l}\text { Interval training: } 2 \text { circuits of } 8 \times 30 \text { s vigorous to } \\
\text { maximal, alternated with } 1 \text { min active rest } \\
\text { Plus } 5 \text { min } 60 \%-75 \% \text { endurance. }\end{array}$ & $\begin{array}{l}\text { Two circuits of 15-20 repetitions. Weights based on } \\
15 \text { RM tests (repeated every } 4 \text { weeks) } \\
\text { Exercises: legs (squat), arms (biceps curl, triceps } \\
\text { extension), shoulder (shoulder press), thorax (barbell } \\
\text { bench press), back (rowing). Abdomen: crunch } 30-40 \\
\text { repetitions; hoover/planking } 2 \times 45 \text { s. }\end{array}$ \\
\hline
\end{tabular}

HRR, heartrate reserve; PAM, Physical Activity and Memory; RM, repetition maximum. 
patient registers the exercise sessions and average heart rate per session in an exercise log, which is monitored by the physiotherapist.

\section{CONTROL GROUP}

Patients in the control group are requested to maintain their habitual physical activity pattern next to the usual care. A supervised exercise programme of 3 months is offered after the study period.

\section{STUDY ENDPOINTS}

At baseline and 6-month follow-up, participants visit the UMC Utrecht for outcome assessments following the same standard operating procedures. At 18-month follow-up, a selection of study endpoint will be assessed.

\section{Baseline and 6-month follow-up measurements Primary outcome}

Primary outcome is the total recall score on the Hopkins Verbal Learning Test-Revised (HVLT-R), a test to assess verbal learning and memory. With this outcome, we also adhere to the guidelines of the International Cognition and Cancer Task Force. ${ }^{56}$ The HVLT-R consists of a 12-word list from three semantic categories, which is read aloud by the researcher. The patient has to recall as many words as possible for three consecutive learning trials (total recall). After $20 \mathrm{~min}$, the patient is asked again to recall as many words as possible (delayed recall). Lastly, a list of 24 words is read by the researcher and the patient is asked to declare if the concerned word was mentioned in the previous list with target words or not (recognition discrimination index). At 6-month follow-up, a parallel version of the 12-word list with different words is used.

\section{Secondary outcomes}

\section{Cognitive functioning}

Self-reported complaints measured by two symptom severity questions on memory and attention and six symptom interference items of the MD Anderson Symptom Inventory for multiple myeloma (MDASI-MM) questionnaire. The MDASI has been proven to be valid, reliable and concise to assess symptoms in patients with cancer. $^{57}$

Overall cognitive functioning is measured by the Amsterdam Cognition Scan, an online neuropsychological test battery which is also performed to select eligible patients. ${ }^{49}$ The neuropsychological test battery includes seven tests including eleven outcome measures and two questionnaires. It contains the following domains: learning and memory, attention and working memory, processing speed, executive functioning and motor functioning. Most tests start with an instruction video and a practice session with feedback. The outcome measures including the corresponding test domains are described in table 1 . This online test battery has shown a high usability and reliability for measuring cognitive functioning. ${ }^{49}$

\section{Neuroradiological assessment}

Changes in brain structure and perfusion are measured by a Philips 3-tesla (T) MRI scanner at the UMC Utrecht. All scans are acquired with a standardised protocol that consists of an axial $\mathrm{T} 2 *_{\text {- }}$ (repetition time in $\mathrm{ms}(\mathrm{TR}) /$ echo time in $\mathrm{ms}(\mathrm{TE})=$ shortest $/ 20), \mathrm{T} 2-/$ proton density-weighted (PD) sequence (TR/TE1/ TE2=3198/19/140) inversion recovery (IR) (TR/TE/ inversion time in $\mathrm{ms} \quad(\mathrm{TI})=4416 / 15 / 400)$, fluid-attenuated inversion recovery (FLAIR) (TR/TE/ $\mathrm{TI}=11000 / 125 / 2800)$, all with 48 contiguous slices and $0.96 \times 0.95 \times 3.00 \mathrm{~mm}^{3}$ voxels, 3D T1-weighted sequence (sagittal acquisition, TR/TE=shortest/4.5; 192 slices; $1.00 \mathrm{~mm}$ isotropic voxels). This allows for multispectra tissue segmentation. Additionally, a Pseudocontinuous Arterial Spin Labelling (pCASL, axial acquisition, TR/ TE $4400 \mathrm{~ms} /$ shortest, 19 slices, $3.0 \times 3.0 \times 7.0 \mathrm{~mm}$ voxels, 30 volumes, M0 images following the pCASL scan with identical readout) is acquired, and a diffusion tensor imaging (DTI) sequence $(\mathrm{TR} / \mathrm{TE}=$ shortest/90, 40 directions, b value 1500,56 slices, $2.50 \mathrm{~mm}$ isotropic voxels). The MRI scan protocol duration is $24 \mathrm{~min}$.

Fully automated brain tissue segmentation is used including segmentation of hippocampus. T1 scans are corrected for intensity inhomogeneity and segmented into different tissue types and subregions including hippocampus. FLAIR is used to determine white matter hyperintensities and for lesion masking. The DTI sequence is used for assessing changes in white matter connectivity/microstructure by calculating the fractional anisotropy). pCASL is added to evaluate changes in brain perfusion.

\section{Patient-reported outcomes}

Sociodemographic data (age, education, marital status), menopausal status, age at menopause, alcohol intake and smoking status are assessed by a self-developed questionnaire.

Fatigue is assessed by the Multidimensional Fatigue Inventory-20. It consists of 20 items including five subscales (general fatigue, physical fatigue, mental fatigue, reduced activity and reduced motivation) to measure multiple fatigue characteristics. ${ }^{58}$

Self-rated anxiety and depression are measured by the Hospital Anxiety and Depression Scale which incorporates 14 items organised in seven questions about anxiety and seven questions about depression. ${ }^{59}$ In addition, depressive symptoms will also be assessed by the Patient Health Questionnaire-9, which has been validated in patients with cancer. ${ }^{60}$

Health-related quality of life (HRQoL) is assessed by the European Organisation Research and Treatment of Cancer-Quality of Life-C30 scale. This validated questionnaire consists of five functional scales (physical, emotional, role, cognitive and social functioning), one quality of life scale and one symptom scale. ${ }^{61}$ Additionally, an overall sum score can be calculated that represents overall HRQoL. ${ }^{62}$ 
Health status is measured using the EuroQol five dimensions and three levels questionnaire consisting of five items with aspect to health status. ${ }^{63}$

Physical activity levels are measured by the short questionnaire to assess health-enhancing physical activity (SQUASH) and an accelerometer. The SQUASH is used to assess habitual physical activity, which includes commuting, leisure time and household activities, and activities at work and/or school. ${ }^{64}$ In addition, all participants wear an accelerometer (Actigraph) for 7 days at the beginning of the intervention period (before the start of the exercise programme) and during the final 7 days of the intervention period.

Sedentary time is assessed by two questions of the International Physical Activity Questionnaires for an estimated sitting time weekly. ${ }^{65}$

Work performance is assessed by the Work Ability Index containing seven items on demand of work, worker's health status and recourses which results in a summary score. ${ }^{6667}$

Anthropometrics (height, weight, waist and hip circumference) are measured in light clothing and without shoes. Body weight is measured on an analogue weighting scale and height by a measuring rod. The body mass index is calculated as the weight in kilograms divided by the height in metre squared $\left(\mathrm{kg} / \mathrm{m}^{2}\right)$. Waist circumference (to the nearest $0.5 \mathrm{~cm}$ ) is measured standing at the smallest circumference between abdomen and chest. Hip circumference (to the nearest $0.5 \mathrm{~cm}$ ) is measured standing as the largest circumference between waist and thigh. Waist and hip circumferences are measured twice and averaged.

Physical fitness is assessed by a sports medicine physician using a CPET with continuous breathing gas analysis at a cycle ergometer. After a 1 min warm-up under no load, the workload increases by 10,15 , or 20 watt, dependent on the patient's condition. The workload increases gradually and the peak workload is reached around $10 \mathrm{~min}$ after the start. The test stops at patients' symptoms or at the physicians' discretions. After the test is terminated, a 3 min cooling-down is performed. Borg scores are taken for fatigue, pain and dyspnoea before and directly after the exercise test. Expired gases and minute ventilation, heart rate and ECG are monitored continuously. Peak workload, peak oxygen uptake and peak heart rate are used for analyses. The peak heart rate achieved on the CPET is also used for determining the HRR to estimate the individual training intensity for patients participating in the exercise programme.

Medical data (date of diagnosis, tumour type, disease stage, type of treatment, comorbidity, endocrine therapy, menopausal status) are retrieved from medical records and The Netherlands Cancer Registry. Medication use is asked at every visit.

Blood sampling is performed to determine biomarkers, such as inflammatory factors and BDNF. Blood samples are stored in the Utrecht Medical Center Utrecht biobank and will be determined in the future.
Adherence to the exercise intervention incorporating both attendance and compliance for the exercise protocol is registered in the exercise logs of the intervention group by the physiotherapist (supervised exercise programme) or the participant (Nordic/power walking).

All adverse events potentially related to the study reported by the patient or observed by the investigator or the physiotherapists are recorded. Serious adverse events are reported to the ethical committee who approved the protocol (Medische Ethical Committee (METC), UMC Utrecht).

\section{Eighteen months follow-up measurements}

Eighteen months after baseline, the online outcome assessments are repeated. These consist of the online neuropsychological test battery and the same patient-reported outcomes used during baseline and 6 months follow-up.

\section{Sample size}

Our primary outcome is the total recall score on the HVLT-R. We consider a change in score from baseline of 5 or more points as a clinical relevant improvement defined by the Reliable Change Index. ${ }^{68} 69$ This test is recommended for assessment of patients with cancer experiencing late cognitive problems and has good sensitivity. ${ }^{4}$ Each patient's total recall score recorded at 6 months is assigned a binary outcome as improvement or failure (stable or declined). A sample size of 73 patients per group is needed to detect an absolute difference of $20 \%$ between groups, considering a Fisher's exact test with a nominal 0.05 two-sided significance level and power of $82 \%$. Taking into account $20 \%$ drop-out, 90 patients per group are needed (total 180). This sample size also achieves $>90 \%$ power to detect a meaningful important difference of 1.0 and 0.9 for 'remembering' and 'paying attention' for self-reported cognitive complaints measured with the MDASI-MM.

\section{Data analysis}

Demographics and other characteristics will be reported descriptively, by treatment group. Means and SD will be calculated for continuous (near-)normally distributed variables and medians and ranges for non-normally distributed variables. Categorical variables will be presented by frequencies per group (absolute numbers and percentages).

For the primary intention-to-treat (ITT) analysis, we will compare the number of patients showing postintervention improvement in the HVLT-R between the intervention group and control group using Fisher's exact tests. The treatment effect will be expressed as an effect estimate with 95\% CIs, adjusted for key prognostic factors (eg, age) and baseline HVLT-R, estimated from log-binomial regression models.

Analysis of covariance (ANCOVA) will be used to analyse the ITT effect on other neuropsychological outcome measures (continuous outcomes of the online 
test battery) and imaging outcomes. The ANCOVA will include the baseline measure and stratification factors as covariates. Complete cases for both baseline and follow-up measurements will be analysed for the different outcomes. ${ }^{70}$ If missing values exceed $10 \%$, we additionally will repeat the analysis by using multiple imputation.

As per protocol analyses, we will repeat the cognitive and imaging analyses for the patients with a minimal adherence of $80 \%$ only. Adherence to the physical therapy training and the Nordic/power walking will be defined as a percentage (the number of attended sessions divided by the number of sessions offered).

Linear regression analysis adjusted for baseline will be used to assess the association between changes in brain structure/function and changes in cognitive functioning. The same analyses will be performed for the association between changes in cognitive functioning and changes in quality of life and work performance.

For the long-term effects, mixed-effect models will be used to analyse the effects of the baseline measurements and two follow-up measurements at 6 and 18 months simultaneously.

\section{Patient and public involvement}

During the development and recruitment phase of the PAM study, two patient advocates advised the study team. A comparable exercise intervention was used in earlier studies in patients with cancer (Physical Activity during Cancer Treatment (PACT) study ${ }^{71}$ ) and a study in postmenopausal women (Sex Hormones And Physical Exercise (SHAPE) II study ${ }^{73}$ ). The burden of the intervention is also assessed by the patient advocates. We will communicate the results of the study to the participating women by a newsletter and a seminar.

\section{Ethics and dissemination}

Ethics

Exercise programmes are recommended in breast cancer survivors as part of care according to multiple guidelines. ${ }^{74-77}$ Physical exercise has beneficial effects on fatigue, HRQoL, anxiety, cardiorespiratory fitness, emotional or perceived physical and social function in patients with breast cancer. ${ }^{78}$ Exercise interventions for cancer survivors are feasible, although achieving high adherence rates appears to be challenging. ${ }^{79}$

Patients who are randomised into the control group have the opportunity to follow a 3-month exercise programme after the study period. Earlier research has shown that patients participating in exercise-oncology studies are motivated to become more physically active and subsequently the risk of contamination between study groups is increased. A systematic review on control group design, contamination and drop-out in exercise-oncology trials showed that control groups receiving an intervention after the study period had lower contamination and drop-out rates. ${ }^{80}$ For this reason and for ethical reasons, we offer patients randomised to the control group an exercise programme after the study period.
This study includes MRI assessments of the brain and a CPET with ECG monitoring, which might involve incidental findings. The majority of the incidental findings will presumably not have clinical consequences and include a wait-and-see policy ${ }^{81}$ In healthy people, the prevalence of incidental findings with clinical consequences on brain MRI scans is $2.7 \%$ and increases with age. ${ }^{82}$ More incidental findings are expected in this study since metastases will happen more frequently in the study population. Furthermore, a previous study showed a higher prevalence of pituitary macroadenomas in patients with breast cancer compared with a reference group. ${ }^{83}$ If an incidental finding is found which is clinically relevant, the participant and her physician will be informed as stated in the informed consent form. Since incidental findings might include metastases, informing patients might result in increased stress. Therefore, particular attention will be given to the quality of communication to the patient.

\section{Dissemination}

To date, no pharmacological intervention exists that can prevent or diminish cognitive problems in patients with non-central nervous system cancer. Physical exercise is a promising non-pharmacological intervention for cognitive decline. If physical exercise appears to be effective, patients with breast cancer with cognitive problems can be offered an evidence-based intervention to reduce these problems and hence improve their quality of life. Furthermore, if effective, this might provide further evidence to support the implementation of routine exercise programmes during cancer treatment with the aim to minimise cognitive problems that might arise.

Since preclinical and human studies have shown that a much wider spectrum of chemotherapy than applied in treatment of breast cancer is associated with cognitive change, we expect that the results of this study will also be applicable for chemotherapy-exposed patients with other solid cancer types with cognitive problems. ${ }^{2}$ Consequently, this study might have a large positive impact on the quality of life of the growing community of (breast) cancer survivors.

\section{Author affiliations}

${ }^{1}$ Julius Center for Health Sciences and Primary Care, University Medical Center Utrecht, Utrecht, The Netherlands

${ }^{2}$ Division of Psychosocial Research and Epidemiology, Netherlands Cancer Institute, Amsterdam, The Netherlands

${ }^{3}$ Department of Medical Oncology, University Medical Center Utrecht, Utrecht, The Netherlands

${ }^{4}$ Center for Quality of Life, Netherlands Cancer Institute, Amsterdam, The Netherlands

${ }^{5}$ ACHIEVE Center of Applied Research, Faculty of Health, University of Applied Sciences, Amsterdam, The Netherlands

${ }^{6}$ Netherlands Comprehensive Cancer Organisation (IKNL), Utrecht, The Netherlands ${ }^{7}$ Medical School Twente, Medisch Spectrum Twente, Enschede, The Netherlands

${ }^{8}$ Department of Research Methodology, Measurement, Universiteit Twente, Enschede, The Netherlands

Contributors EMM, SBS, AMM, MBdR, MIG and PHMP initiated the collaborative project and together with LW and EWK the trial is executed and coordinated. MBdR, MIG, PHMP, EWK, EvdW, MS, GS, MJV, JAMvdP and JJJ provided clinical, 
neuropsychological, neuroimaging and physiological expertise. LW wrote the first draft of this manuscript. All authors contributed and approved the final version of the manuscript for publication. We would like to thank the patient advocates, Yve Brouwers and Cornelie Bierens de Haan, who contributed to the design and conduction of the PAM study.

Funding This work is supported by KWF kankerbestrijding, grant number UU 2015-7954.

Competing interests None declared.

Patient consent for publication Not required.

Ethics approval The Medical Ethics Committee of the University Medical Center (UMC) Utrecht approved the study.

Provenance and peer review Not commissioned; externally peer reviewed.

Open access This is an open access article distributed in accordance with the Creative Commons Attribution Non Commercial (CC BY-NC 4.0) license, which permits others to distribute, remix, adapt, build upon this work non-commercially, and license their derivative works on different terms, provided the original work is properly cited, appropriate credit is given, any changes made indicated, and the use is non-commercial. See: http://creativecommons.org/licenses/by-nc/4.0/.

\section{REFERENCES}

1. Ahles TA, Root JC, Ryan EL. Cancer- and cancer treatmentassociated cognitive change: an update on the state of the science. $J$ Clin Oncol 2012;30:3675-86.

2. Wefel JS, Kesler SR, Noll KR, et al. Clinical characteristics, pathophysiology, and management of noncentral nervous system cancer-related cognitive impairment in adults. CA Cancer J Clin 2015;65:123-38.

3. Jenkins V, Shilling V, Deutsch G, et al. A 3-year prospective study of the effects of adjuvant treatments on cognition in women with early stage breast cancer. Br J Cancer 2006;94:828-34.

4. Wefel JS, Saleeba AK, Buzdar AU, et al. Acute and late onset cognitive dysfunction associated with chemotherapy in women with breast cancer. Cancer 2010;116:3348-56.

5. Janelsins MC, Kesler SR, Ahles TA, et al. Prevalence, mechanisms, and management of cancer-related cognitive impairment. Int Rev Psychiatry 2014;26:102-13.

6. de Ruiter MB, Reneman L, Boogerd W, et al. Late effects of highdose adjuvant chemotherapy on white and gray matter in breast cancer survivors: converging results from multimodal magnetic resonance imaging. Hum Brain Mapp 2012;33:2971-83.

7. Koppelmans V, Breteler MM, Boogerd W, et al. Neuropsychological performance in survivors of breast cancer more than 20 years after adjuvant chemotherapy. J Clin Oncol 2012;30:1080-6.

8. Mitchell AJ, Kemp S, Benito-León J, et al. The influence of cognitive impairment on health-related quality of life in neurological disease. Acta Neuropsychiatr 2010;22:2-13.

9. Dietrich J, Han R, Yang Y, et al. CNS progenitor cells and oligodendrocytes are targets of chemotherapeutic agents in vitro and in vivo. J Biol 2006;5:22.

10. Han R, Yang YM, Dietrich J, et al. Systemic 5-fluorouracil treatment causes a syndrome of delayed myelin destruction in the central nervous system. J Biol 2008;7:12.

11. Seigers R, Schagen SB, Van Tellingen O, Van TO, et al. Chemotherapy-related cognitive dysfunction: current animal studies and future directions. Brain Imaging Behav 2013;7:453-9.

12. Li M, Caeyenberghs K. Longitudinal assessment of chemotherapyinduced changes in brain and cognitive functioning: A systematic review. Neurosci Biobehav Rev 2018;92:304-17.

13. Stouten-Kemperman MM, de Ruiter MB, Koppelmans V, et al. Neurotoxicity in breast cancer survivors $\geq 10$ years post-treatment is dependent on treatment type. Brain Imaging Behav 2015;9:275-84.

14. Koppelmans V, de Ruiter MB, van der Lijn F, et al. Global and focal brain volume in long-term breast cancer survivors exposed to adjuvant chemotherapy. Breast Cancer Res Treat 2012;132:1099-106.

15. Pomykala KL, de Ruiter MB, Deprez S, et al. Integrating imaging findings in evaluating the post-chemotherapy brain. Brain Imaging Behav 2013;7:436-52.

16. McDonald BC, Saykin AJ. Alterations in brain structure related to breast cancer and its treatment: chemotherapy and other considerations. Brain Imaging Behav 2013;7:374-87.

17. Menning S, de Ruiter MB, Veltman DJ, et al. Changes in brain white matter integrity after systemic treatment for breast cancer: a prospective longitudinal study. Brain Imaging Behav 2018;12:324-34.
18. Stouten-Kemperman MM, de Ruiter MB, Caan MW, et al. Lower cognitive performance and white matter changes in testicular cancer survivors 10 years after chemotherapy. Hum Brain Mapp 2015;36:4638-47.

19. Deprez S, Billiet T, Sunaert S, et al. Diffusion tensor MRI of chemotherapy-induced cognitive impairment in non-CNS cancer patients: a review. Brain Imaging Behav 2013;7:409-35.

20. Chen X, He X, Tao L, et al. The attention network changes in breast cancer patients receiving neoadjuvant chemotherapy: Evidence from an arterial spin labeling perfusion study. Sci Rep $2017 ; 7: 42684$

21. Nudelman KN, Wang $Y$, McDonald BC, et al. Altered cerebral blood flow one month after systemic chemotherapy for breast cancer: a prospective study using pulsed arterial spin labeling MRI perfusion. PLoS One 2014;9:e96713.

22. Deprez S, Vandenbulcke M, Peeters R, et al. Longitudinal assessment of chemotherapy-induced alterations in brain activation during multitasking and its relation with cognitive complaints. J Clin Oncol 2014;32:2031-8.

23. Carvalho A, Rea IM, Parimon T, et al. Physical activity and cognitive function in individuals over 60 years of age: a systematic review. Clin Interv Aging 2014;9:661-82.

24. Smith PJ, Blumenthal JA, Hoffman BM, et al. Aerobic exercise and neurocognitive performance: a meta-analytic review of randomized controlled trials. Psychosom Med 2010;72:239-52.

25. Klusmann V, Evers A, Schwarzer R, et al. Complex mental and physical activity in older women and cognitive performance: a 6-month randomized controlled trial. J Gerontol A Biol Sci Med Sci 2010;65:680-8.

26. Nagamatsu LS, Handy TC, Hsu CL, et al. Resistance training promotes cognitive and functional brain plasticity in seniors with probable mild cognitive impairment. Arch Intern Med 2012;172:666-8.

27. Baker LD, Frank LL, Foster-Schubert K, et al. Effects of aerobic exercise on mild cognitive impairment: a controlled trial. Arch Neurol 2010;67:71-9.

28. Lazarov O, Mattson MP, Peterson DA, et al. When neurogenesis encounters aging and disease. Trends Neurosci 2010;33:569-79.

29. Ainslie PN, Cotter JD, George KP, et al. Elevation in cerebral blood flow velocity with aerobic fitness throughout healthy human ageing. $J$ Physiol 2008;586:4005-10.

30. Lojovich JM. The relationship between aerobic exercise and cognition: is movement medicinal? J Head Trauma Rehabil 2010;25:184-92

31. Meneses-Echávez JF, Correa-Bautista JE, González-Jiménez E, et al The effect of exercise training on mediators of inflammation in breast cancer survivors: A systematic review with meta-analysis. Cancer Epidemiol Biomarkers Prev 2016;25:1009-17.

32. Kang DW, Lee J, Suh SH, et al. Effects of Exercise on Insulin, IGF Axis, Adipocytokines, and Inflammatory Markers in Breast Cancer Survivors: A Systematic Review and Meta-analysis. Cancer Epidemiol Biomarkers Prev 2017;26:355-65.

33. Knaepen K, Goekint M, Heyman EM, et al. Neuroplasticity - exerciseinduced response of peripheral brain-derived neurotrophic factor: a systematic review of experimental studies in human subjects. Sports Med 2010;40:765-801.

34. Barde YA. Neurotrophins: a family of proteins supporting the survival of neurons. Prog Clin Biol Res 1994;390:45-56.

35. Leibrock J, Lottspeich F, Hohn A, et al. Molecular cloning and expression of brain-derived neurotrophic factor. Nature 1989;341:149-52.

36. Dinoff A, Herrmann N, Swardfager W, et al. The effect of exercise training on resting concentrations of peripheral brainderived neurotrophic factor (bdnf): A meta-analysis. PLoS One 2016;11:e0163037.

37. Vaynman S, Ying Z, Gomez-Pinilla F. Hippocampal BDNF mediates the efficacy of exercise on synaptic plasticity and cognition. Eur J Neurosci 2004;20:2580-90.

38. Erickson KI, Voss MW, Prakash RS, et al. Exercise training increases size of hippocampus and improves memory. Proc Natl Acad Sci U S A 2011;108:3017-22.

39. ten Brinke LF, Bolandzadeh N, Nagamatsu LS, et al. Aerobic exercise increases hippocampal volume in older women with probable mild cognitive impairment: a 6-month randomised controlled trial. $\mathrm{Br} J$ Sports Med 2015;49.

40. Colcombe SJ, Erickson KI, Scalf PE, et al. Aerobic exercise training increases brain volume in aging humans. J Gerontol A Biol Sci Med Sci 2006;61:1166-70.

41. Chapman SB, Aslan S, Spence JS, et al. Shorter term aerobic exercise improves brain, cognition, and cardiovascular fitness in aging. Front Aging Neurosci 2013;5:75. 
42. Zimmer P, Baumann FT, Oberste M, et al. Effects of Exercise Interventions and Physical Activity Behavior on Cancer Related Cognitive Impairments: A Systematic Review. Biomed Res Int 2016;2016:1-13.

43. Campbell KL, Kam JWY, Neil-Sztramko SE, et al. Effect of aerobic exercise on cancer-associated cognitive impairment: A proof-ofconcept RCT. Psychooncology 2018;27.

44. Hartman SJ, Nelson SH, Myers E, et al. Randomized controlled trial of increasing physical activity on objectively measured and selfreported cognitive functioning among breast cancer survivors: The memory \& motion study. Cancer 2018;124:192-202.

45. Baumann FT, Drosselmeyer N, Leskaroski A, et al. 12-Week Resistance Training with Breast Cancer Patients during Chemotherapy: Effects on Cognitive Abilities. Breast Care 2011;6:142-3.

46. Schmidt ME, Wiskemann J, Armbrust P, et al. Effects of resistance exercise on fatigue and quality of life in breast cancer patients undergoing adjuvant chemotherapy: A randomized controlled trial. Int J Cancer 2015;137:471-80.

47. Steindorf K, Schmidt ME, Klassen O, et al. Randomized, controlled trial of resistance training in breast cancer patients receiving adjuvant radiotherapy: results on cancer-related fatigue and quality of life. Ann Oncol 2014;25:2237-43.

48. Buwalda B, Schagen SB. Is basic research providing answers if adjuvant anti-estrogen treatment of breast cancer can induce cognitive impairment? Life Sci 2013;93:581-8.

49. Feenstra HEM, Murre JMJ, Vermeulen IE, et al. Reliability and validity of a self-administered tool for online neuropsychological testing: The Amsterdam Cognition Scan. J Clin Exp Neuropsychol 2018;40:1-21.

50. Feenstra HE, Vermeulen IE, Murre JM, et al. Online cognition: factors facilitating reliable online neuropsychological test results. Clin Neuropsychol 2017;31:59-84.

51. Gehring K, Sitskoorn MM, Gundy CM, et al. Cognitive rehabilitation in patients with gliomas: a randomized, controlled trial. J Clin Oncol 2009;27:3712-22.

52. Willson J, Torry MR, Decker MJ, et al. Effects of walking poles on lower extremity gait mechanics. Med Sci Sports Exerc 2001;33:142-7.

53. Church TS, Earnest CP, Morss GM. Field testing of physiological responses associated with Nordic Walking. Res Q Exerc Sport 2002;73:296-300.

54. Skórkowska-Telichowska K, Kropielnicka K, Bulińska K, et al. Nordic walking in the second half of life. Aging Clin Exp Res 2016;28:1035-46.

55. Kim C, Kim BO, Lim KB, et al. The effect of power-walking in phase 2 cardiac rehabilitation program. Ann Rehabil Med 2012;36:133-40.

56. Wefel JS, Vardy J, Ahles T, et al. International Cognition and Cancer Task Force recommendations to harmonise studies of cognitive function in patients with cancer. Lancet Oncol 2011;12:703-8.

57. Jones D, Vichaya EG, Wang XS, et al. Acute cognitive impairment in patients with multiple myeloma undergoing autologous hematopoietic stem cell transplant. Cancer 2013;119:4188-95.

58. Smets EM, Garssen B, Bonke B, et al. The Multidimensional Fatigue Inventory (MFI) psychometric qualities of an instrument to assess fatigue. J Psychosom Res 1995;39:315-25.

59. Osborne RH, Elsworth GR, Sprangers MA, et al. The value of the Hospital Anxiety and Depression Scale (HADS) for comparing women with early onset breast cancer with population-based reference women. Qual Life Res 2004;13:191-206.

60. Hinz A, Mehnert A, Kocalevent RD, et al. Assessment of depression severity with the PHQ-9 in cancer patients and in the general population. BMC Psychiatry 2016;16:22-728.

61. Aaronson NK, Ahmedzai S, Bergman B, et al. The European Organization for Research and Treatment of Cancer QLQ-C30: a quality-of-life instrument for use in international clinical trials in oncology. J Natl Cancer Inst 1993;85:365-76.

62. Giesinger JM, Kieffer JM, Fayers PM, et al. Replication and validation of higher order models demonstrated that a summary score for the EORTC QLQ-C30 is robust. J Clin Epidemiol 2016;69:79-88.
63. Pickard AS, Wilke CT, Lin HW, et al. Health utilities using the EQ-5D in studies of cancer. Pharmacoeconomics 2007;25:365-84.

64. Wendel-Vos GC, Schuit AJ, Saris WH, et al. Reproducibility and relative validity of the short questionnaire to assess health-enhancing physical activity. J Clin Epidemiol 2003;56:1163-9.

65. Craig CL, Marshall AL, Sjöström M, et al. International physical activity questionnaire: 12-country reliability and validity. Med Sci Sports Exerc 2003;35:1381-95.

66. Ilmarinen J, Rantanen J. Promotion of work ability during ageing. Am $J$ Ind Med 1999;36:21-3.

67. de Zwart BCH, Frings-Dresen MH, van Duivenbooden JC. Testretest reliability of the Work Ability Index questionnaire. Occup Med 2002;52:177-81.

68. Jacobson NS, Truax P. Clinical significance: A statistical approach to defining meaningful change in psychotherapy research. $J$ Consult Clin Psychol 1991;59:12-19.

69. Chang EL, Wefel JS, Hess KR, et al. Neurocognition in patients with brain metastases treated with radiosurgery or radiosurgery plus whole-brain irradiation: a randomised controlled trial. Lancet Oncol 2009;10:1037-44.

70. Groenwold RHH, Donders ART, Roes KCB, et al. Dealing With Missing Outcome Data in Randomized Trials and Observational Studies. Am J Epidemiol 2012;175:210-7.

71. Travier N, Velthuis MJ, Steins Bisschop CN, et al. Effects of an 18-week exercise programme started early during breast cancer treatment: a randomised controlled trial. BMC Med 2015;13:121-362.

72. van Vulpen JK, Velthuis MJ, Steins Bisschop CN, et al. Effects of an Exercise Program in Colon Cancer Patients undergoing Chemotherapy. Medicine \& Science in Sports \& Exercise 2016;48:767-75.

73. van Gemert WA, May AM, Schuit AJ, et al. Effect of Weight Loss with or without Exercise on Inflammatory Markers and Adipokines in Postmenopausal Women: The SHAPE-2 Trial, A Randomized Controlled Trial. Cancer Epidemiology Biomarkers \& Prevention 2016;25:799-806.

74. van den Berg JP, Velthuis MJ, Gijsen BC, et al. Guideline "Cancer rehabilitation"]. Ned Tijdschr Geneeskd 2011;155:A4104.

75. Hayes SC, Spence RR, Galvão DA, et al. Australian Association for Exercise and Sport Science position stand: Optimising cancer outcomes through exercise. J Sci Med Sport 2009;12:428-34

76. Schmitz KH, Courneya KS, Matthews C, et al. American College of Sports Medicine Roundtable on Exercise Guidelines for Cancer Survivors. Medicine \& Science in Sports \& Exercise 2010;42:1409-26.

77. Buffart LM, Galvão DA, Brug J, et al. Evidence-based physical activity guidelines for cancer survivors: Current guidelines, knowledge gaps and future research directions. Cancer Treat Rev 2014:40:327-40.

78. Lahart IM, Metsios GS, Nevill AM, et al. Physical activity for women with breast cancer after adjuvant therapy. Cochrane Database Syst Rev 2018;1:CD011292.

79. Kampshoff CS, Jansen F, van Mechelen W, van MW, et al. Determinants of exercise adherence and maintenance among cancer survivors: a systematic review. Int J Behav Nutr Phys Act 2014;11:80-11.

80. Steins Bisschop CN, Courneya KS, Velthuis MJ, et al. Control Group Design, Contamination and Drop-Out in Exercise Oncology Trials: A Systematic Review. PLoS One 2015;10:e0120996.

81. Bos D, Poels MMF, Adams HHH, et al. Prevalence, clinical management, and natural course of incidental findings on brain $\mathrm{mr}$ images: The population-based rotterdam scan study. Radiology 2016;281:507-15.

82. Morris Z, Whiteley WN, Longstreth WT, et al. Incidental findings on brain magnetic resonance imaging: systematic review and metaanalysis. BMJ 2009;339:b3016.

83. Koppelmans V, Schagen SB, Poels MMF, et al. Incidental findings on brain Magnetic Resonance Imaging in long-term survivors of breast cancer treated with adjuvant chemotherapy. Eur $J$ Cancer 2011;47:2531-6. 